\title{
Triphenylmethanol Conjugates of Triptorelin as Anti-Lipid Peroxidation Prodrugs
}

\author{
Samiyah Alhamed, Jawzah Alnakhli, William Boadi, Ryan Beni* \\ Department of Chemistry, Tennessee State University, Nashville TN, USA \\ Email: ^rbeni@tnstate.edu
}

How to cite this paper: Alhamed, S., Alnakhli, J., Boadi, W. and Beni, R. (2019) Triphenylmethanol Conjugates of Triptorelin as Anti-Lipid Peroxidation Prodrugs. Open Journal of Medicinal Chemistry, 9, 49-62.

https://doi.org/10.4236/ojmc.2019.93003

Received: June 18, 2019

Accepted: July 23, 2019

Published: July 26, 2019

Copyright $\odot 2019$ by author(s) and Scientific Research Publishing Inc. This work is licensed under the Creative Commons Attribution International License (CC BY 4.0).

http://creativecommons.org/licenses/by/4.0/

\begin{abstract}
Antioxidants are substances that can prevent or slow damage to cells caused by free radicals, unstable molecules that the body produces as a reaction to environmental and other pressures. Free radicals may play a role in heart disease, cancer and other diseases. If the body cannot process and remove free radicals efficiently, oxidative stress can result. This can harm cells and body function. Free radicals are also known as reactive oxygen species (ROS). In this research, Triptorelin ${ }^{\circledR}$ (TRP) conjugates of triphenylmethanol derivatives (TPMs) were synthesized to evaluate their in vitro lipid peroxidation potency. Comparative lipid peroxidation assays between TRP-TPMs conjugates and the corresponding TPMs derivatives were measured using thiobarbituric reactive substance (TBARS) in a dose- and time-dependent manner following the Fenton's pathway. Overall, TBARS decreased between $20 \%-30 \%$ for the treated samples of synthesized conjugates compared to their respective control physical mixtures. These data suggest that TRP-TPMs derivatives can be used to improve the biological activity of TRP.
\end{abstract}

\section{Keywords}

Antioxidants, Prodrugs, Triptorelin, Polyphenols, Lipid Peroxidation,

Triphenylmethanol

\section{Introduction}

Triptorelin ${ }^{\circledR}$ (TRP) is a synthetic analogue of the gonadotropin-releasing hormone $(\mathrm{GnRH})$, first reported in 1976. The structure of TRP consists of ten amino acids (5-oxoPro-His-Trp-Ser-Tyr-D-Trp-Leu-Arg-Pro-GlyNH ${ }_{2}$ ) and is used to treat advanced prostate cancer and endometriosis. Initially TRP stimulates the pituitary secretion of FSH and LH, however, prolonged stimulation (constant concentration of TRP in the blood) of the pituitary causes insensitive to the ac- 
tion of GnRH. This reduces the level of gonadotropin in the blood, resulting in decreased levels of sex hormones to postcastration or menopausal levels. These effects are reversible. In addition to the usual side effects of the agonist analogs of LH-RH, other reported adverse effects include transient hypertension, dry mouth, excessive salivation, paraesthesia and increased dysuria [1].

LHRH analogues are found in a variety of formulations and depending on the medication, can be administered every 1 to 12 months. Medications currently available in the United States include different formulations of triptorelin ${ }^{\circledR}$ leuprolide $^{\circledR}$, goserelin ${ }^{\circledR}$ and histrelin ${ }^{\circledR}$ in a variety of dosing intervals ranging from monthly to yearly. The associated side effects include hot flashes, decreased libido, erectile dysfunction, loss of bone mineral density, anemia and mood changes [2].

Efficacy and toxicity of anticancer drugs can be modified by using drug delivery systems and adjusting the physicochemical properties such as lipophilicity, cellular uptake and prolonging activity through chemical conjugation with various chemical moieties. Drug delivery systems avoid the P-glycoprotein and other multidrug resistance proteins (MRPs) that are involved in drug efflux to overcome the resistance problem and P-glycoprotein-mediated drug efflux [3] [4] [5].

Prodrug strategy is a drug delivery system through which chemical conjugation with the parent drug [6] [7] has been widely used in the delivery of anticancer drugs such as Doxorubicin ${ }^{\circledR}$ [8] [9]. For example, several conjugation methods have been used to improve the delivery of Doxorubicin ${ }^{\circledR}$, including using gold nanoparticles [10], gold nanospheres [11], liposomes [12], peptides [13]-[18] and dendrimers [19]. The conjugation of TRP with agents that have optimal lipophilicity has yet to be explored. Therefore, the development of efficient and safe prodrug carriers to enhance the delivery and retention of TRP into drug-resistant tumor cells remains less explored.

Polyphenols are naturally occurring compounds found largely in fruits, vegetables, cereals and beverages. Fruits like grapes, apples, pears, cherries and berries contain up to $200-300 \mathrm{mg}$ of polyphenols per 100 grams fresh weight [20] [21] [22]. In the last decade, there has been much interest in the potential health benefits of dietary plant polyphenols as antioxidants. The effect of polyphenols on human cancer cell lines is most often protective and induces a reduction in the number of tumors or rate of growth. These effects have been observed at various sites including the mouth, stomach, duodenum, colon, liver, lungs, mammary glands and skin. Many polyphenols, such as quercetin, catechins, isoflavones, lignans, flavanones, ellagic acid, red wine polyphenols, resveratrol and curcumin have been tested; all of them showed protective effects in some models although their mechanisms of action were found to be different [23] [24]. Polyphenols influence the metabolism of pro-carcinogens by modulating the expression of cytochrome P450 enzymes involved in their activation of carcinogens [25] [26].

To take advantage of the antioxidant properties of polyphenolic antioxidants, several polyphenolic derivatives were chosen for the chemical modification of 
TRP. Dodecanedioic acid has been chosen as a lipophilic linker to attach TRP to polyphenolic derivatives.

In this study, we first report the synthesis of the antioxidant triphenylmethanol (TPMs) derivatives of TRP through the covalent conjugation with dodecanedioic acid as the linker. Second, we report the evaluation of their in vitro lipid peroxidation activities in cell lines. Prodrug conjugates were designed to improve cellular uptake, prolong biological activity and reduce therapeutic dosage of TRP. This study examines the antioxidant potential (ROS scavenging action) of TRP conjugate as a measure of their efficacy.

\section{Experimental}

TRP, DMSO and other chemicals and reagents were purchased from Fisher Scientific or Sigma-Aldrich Chemical Co. All coupling reactions (Figure 1) were carried out in Bio-Rad polypropylene columns by shaking and mixing using a Glass $\mathrm{Col}^{\circledR}$ small tube rotator in dry conditions at room temperature. TPMs 1a-c which are the mimics of natural poly phenolic antioxidants, were synthesized in moderate yield (Figure 1) for covalent attachment to TRP via a hydrophobic linker, dodecanedioic acid. Tris(2-(hydroxymethyl) phenol) conjugates of TRP (2a-c) with optimal hydrophobicity were synthesized to carry TRP into the cells (Figure 1). All products were purified ( $\leq 95 \%)$ by a flash chromatography system (TeledyneCombiFlash ${ }^{\circledR}$ Rf-200) and the structures of all the final compounds were confirmed by ESI/TOF mass spectrometry. Figure 2 depicts the structure of the final TRP-TPMs conjugates $2 \mathrm{a}-\mathbf{c}$. $^{1}$

\subsection{Preparation of TPMs 1a-c Derivatives}

The TPMs were synthesized using a modified method based on literature [27] [28], (Figure 1). Accordingly, 1,3,5-trioxane (15 mmole) was added to 2-fluoroanisole, 2-notroanisole or 2-methylanisole (100 mmole) in $10 \mathrm{~mL}$ glacial acetic acid. The mixture was heated to $90^{\circ} \mathrm{C}-95^{\circ} \mathrm{C}$, then $1 \mathrm{~mL}$ of sulfuric acid: glacial acetic acid $(1: 5, \mathrm{v} / \mathrm{v})$ was added to the solution. The mixture was stirred for $5 \mathrm{~h}$ at $90^{\circ} \mathrm{C}-$ $96^{\circ} \mathrm{C}$. The reaction mixture was then cooled to $0^{\circ} \mathrm{C}$ using an ice bath and a homogenous solution of sodium nitrite $(1.0 \mathrm{~g}, 15 \mathrm{mmole})$ and 2 -fluoroanisole, 2-notroanisole or 2-methylanisole ( $15 \mathrm{mmole})$ in $10 \mathrm{ml}$ concentrated sulfuric acid was added to the reaction mixture. The ice bath was removed and stirring continued at room temperature for an additional $24 \mathrm{hr}$ the mixture was then poured into crushed ice (100 g) while stirring. The precipitate was filtered off and dried under vacuum and further purified on $\mathrm{C}_{18}$ column and hexanes/ethyl acetate as solvent using a TeledyneCombiFlash ${ }^{\circledR}$ Rf-200 chromatography machine with the gradient system set at a constant flow rate of $25 \mathrm{ml} / \mathrm{min}$ to yield pure products in $59 \%-81 \%$

\footnotetext{
${ }^{1}$ There is a competition between phenol and primary alcohol to form ester with the linker. The alcohol has higher nucleophilicity in this reaction condition, however its nucleophilicity hampers by higher steric hindrance. Since the phenol is not the phenolate form, it is less reactive than alcohols for nucleophilic reactions, in contrast the lower steric hindrance around the phenol increases its nucleophilicity. Therefore, a mixture of esters is expected. We were not able to separate the mixture by chromatography technique.
} 

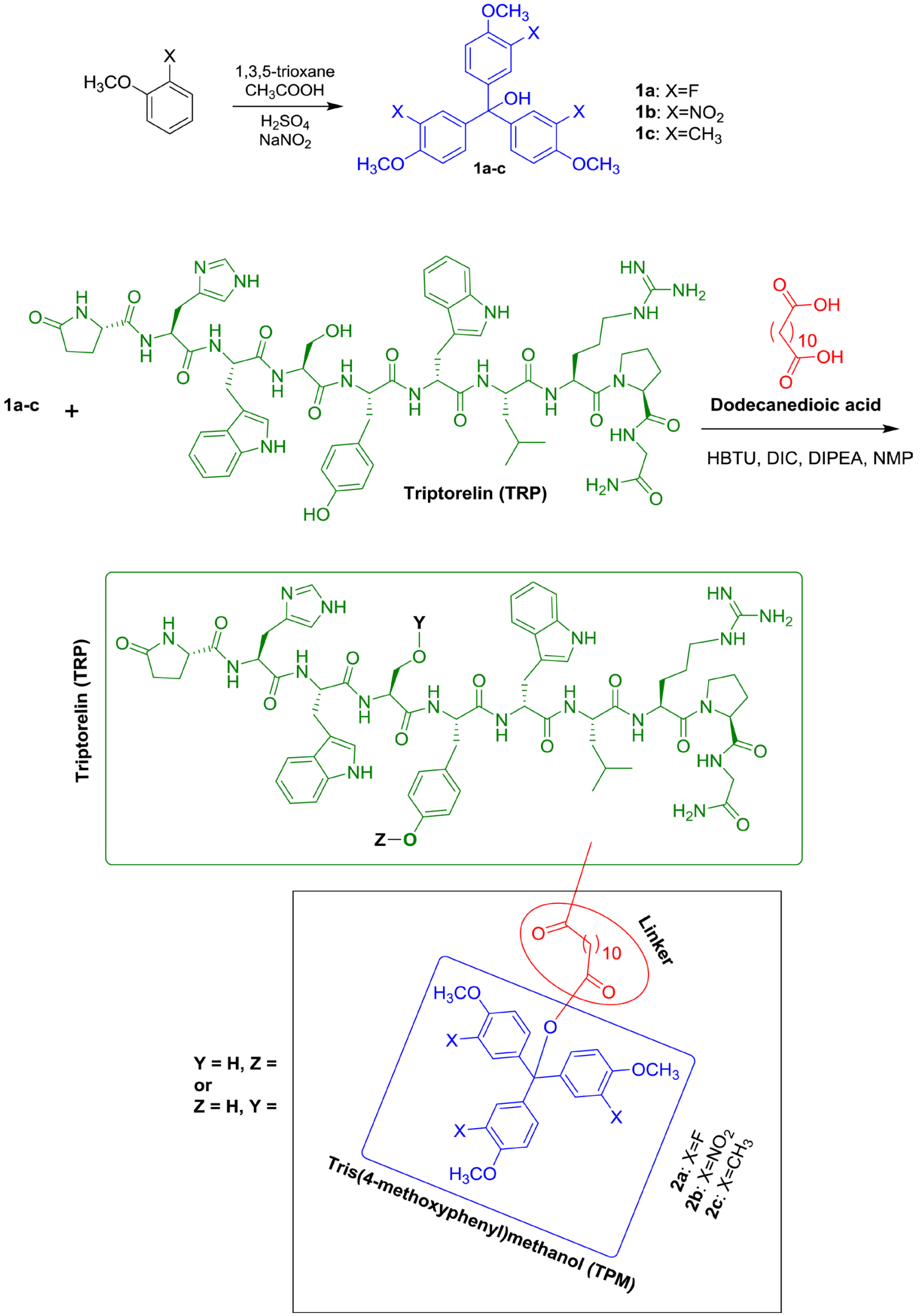

Figure 1. Synthesis of triphenylmethanol (TPMs) derivatives 1a-c and triphenylmethanol conjugates of TRP 2a-c. 


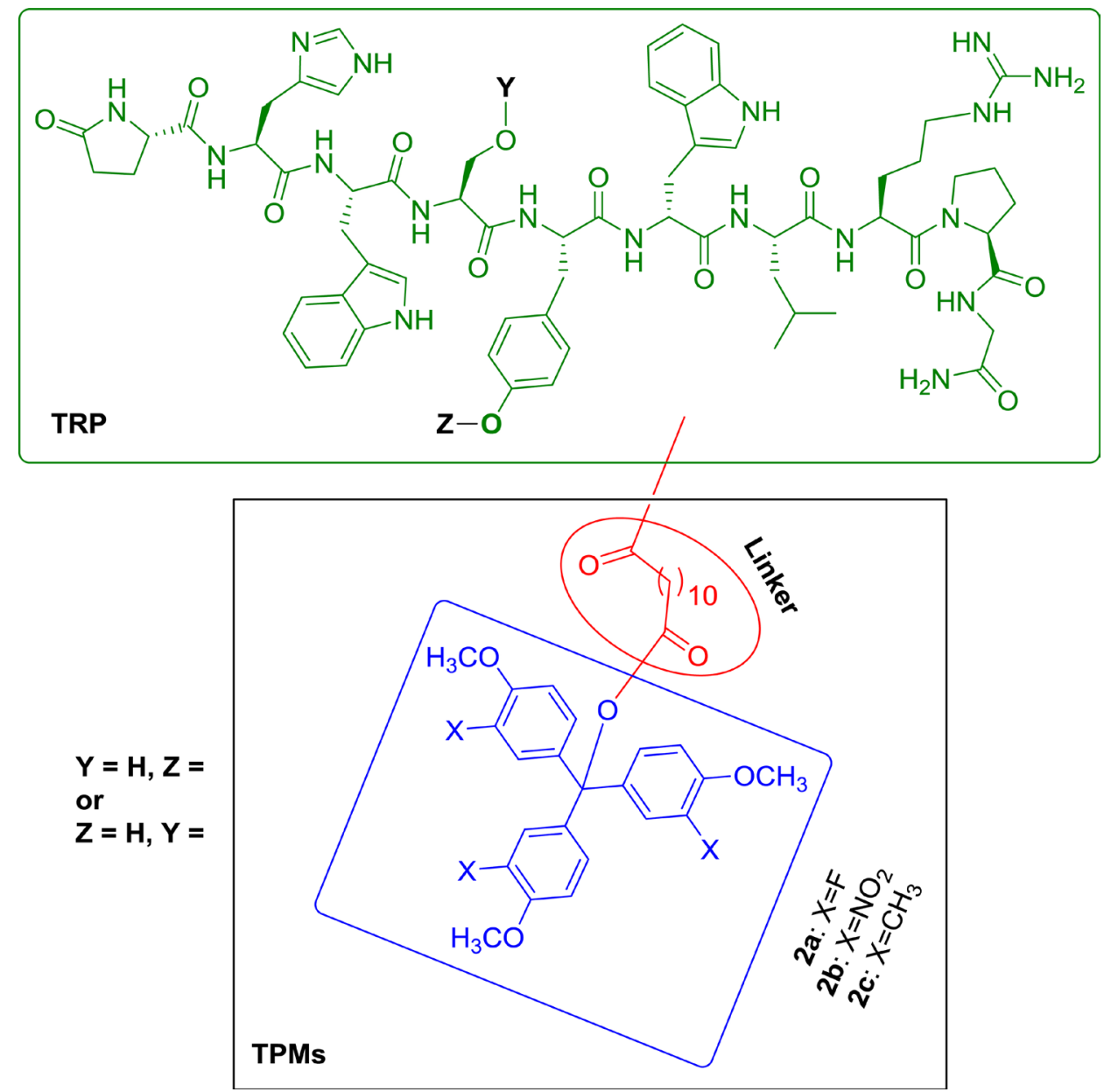

Figure 2. Structure of synthesized TRP-TPMs derivatives 2a-c.

yield. Tris(3-fluoro-4-methoxyphenyl)methanol (1a), (3.59 g, 59\%), MS (ESI-TOF) $(\mathrm{m} / \mathrm{z})$ for $\mathrm{C}_{22} \mathrm{H}_{19} \mathrm{~F}_{3} \mathrm{O}_{4}$ : calcd., 404.1, found 404.1 [M] $]^{+}$; tris(3-nitro-4-methoxyphenyl) methanol (1b), (4.52 g, 63\%), MS (ESI-TOF) $(\mathrm{m} / \mathrm{z})$ for $\mathrm{C}_{22} \mathrm{H}_{19} \mathrm{~N}_{3} \mathrm{O}_{10}$ : calcd, 485.1, found $485.2[\mathrm{M}]^{+}$; tris(3-methyl-4-methoxyphenyl)methanol (1c), (4.77 g, 81\%), MS (ESI-TOF) (m/z) for $\mathrm{C}_{25} \mathrm{H}_{28} \mathrm{O}_{4}$ : calcd. 392.2, found $392.1[\mathrm{M}]^{+}$.

\subsection{Preparation of Tris(2-(Hydroxymethyl) Phenol) Conjugates of TRP 2a-c}

Tris(4-methoxyphenyl)methanol derivatives 1a-c (0.05 mmol), TRP acetate (0.05 mmol), dodecanedioic acid, $12 \mathrm{mg}, 0.05 \mathrm{mmol}$ ) and HBTU (19 mg, 0.05 $\mathrm{mmol}$ ) were dissolved in dry NMP (3 mL). N,N'-diisopropylcarbodiimide (DIC, $8 \mu \mathrm{L}, 0.05 \mathrm{mmol}$ ) and N,N-Diisopropylethylamine (DIPEA, $21 \mu \mathrm{L}, 0.12 \mathrm{mmol}$ ) were added to the reaction mixture. The mixture was stirred at room temperature for $24 \mathrm{~h}$. Afterwards, the solvent was evaporated and dried under vacuum. The final product was purified with a $\mathrm{C}_{18}$ column and hexanes/ethyl acetate as solvents using a TeledyneCombiFlash ${ }^{\circledR}$ Rf-200 chromatography machine. The gradient system set at a constant flow rate of $25 \mathrm{ml} / \mathrm{min}$ to yield pure TRP-TPMs conjugates 2a-c (Figure 1). TRP-TPMs conjugate (2a), (61 mg, 62\%), MS (ESI-TOF) 
$(\mathrm{m} / z)$ for $\mathrm{C}_{98} \mathrm{H}_{120} \mathrm{~F}_{3} \mathrm{~N}_{18} \mathrm{O}_{19}$ : calcd, 1908.8, found 1908.7 [M + H] $]^{+}$; TRP-TPMs conjugate (2b), (57 mg, 54\%), MS (ESI-TOF) $(\mathrm{m} / z)$ for $\mathrm{C}_{98} \mathrm{H}_{120} \mathrm{~N}_{21} \mathrm{O}_{25}$ : calcd, 1989.9, found $1989.8[\mathrm{M}+\mathrm{H}]^{+}$; TRP-TPMs conjugate $(2 \mathrm{c}),(67 \mathrm{mg}, 65 \%)$, MS (ESI-TOF) $(\mathrm{m} / z)$ for $\mathrm{C}_{101} \mathrm{H}_{129} \mathrm{~N}_{18} \mathrm{O}_{19}$ : calcd, 1897.0, found $1896.9[\mathrm{M}+\mathrm{H}]^{+}$.

\subsection{Antioxidant Evaluation of TRP-TPMs on Lipid Peroxidation.}

The amount of $3 \mu \mathrm{l}$ Canola vegetable oil in a total reaction volume of $1000 \mu \mathrm{l}$ consistent of $0.05 \mathrm{M}$ Tris- $\mathrm{HCl} \mathrm{pH} \mathrm{7.4,} \mathrm{containing} 0.15 \mathrm{M} \mathrm{KCl}$, and $0.02 \%$ Sodium dodecyl phosphate (SDS), to which was added to a freshly prepared solution of $5 \mu \mathrm{l}$ hydrogen peroxide $\left(\mathrm{H}_{2} \mathrm{O}_{2}\right)$ and $\mathrm{Fe}^{2+}$ (as Ferrous sulphate $\left(\mathrm{FeSO}_{4} .7 \mathrm{H}_{2} \mathrm{O}\right)$ respectively. To different aliquots of this solution each containing, $5 \mu \mathrm{H}_{2} \mathrm{O}_{2}, 5 \mu \mathrm{l}$ of $\mathrm{Fe}^{2+}$ and $3 \mu \mathrm{l}$ of the vegetable oil in Tris buffer, progressively increasing concentrations of different triptorelin derivatives were added. The samples were incubated at $37^{\circ} \mathrm{C}$ for 4,8 and $24 \mathrm{hr}$ Control consisted of all the above reagents except the triptorelin derivatives.

\subsection{Analysis of the Lipid Peroxidation Compounds in the Samples}

2-Thiobarbituric acid (TBA, $2 \mathrm{ml}$ of $67 \%$ solution), was added to the above samples and incubated at $60^{\circ} \mathrm{C}$ for $1 \mathrm{hr}$ in a water bath. The samples were then cooled down to room temperature and $2 \mathrm{ml}$ of n-butanol was added. Then $220 \mu \mathrm{l}$ each of the upper butanol layer was transferred into a-96 well plate. Absorbance was measured at $532 \mathrm{~nm}$ in a Synergy HT Microplate Reader (Winooski, VT).

\subsection{Calculation of Lipid Peroxides in the Samples}

The levels of lipid peroxide were calculated as thiobarbituric acid reactive substances (TBARS) using a molar extinction coefficient or molar absorptivity for the TBA $\left(1.65 \times 10^{-3} \mathrm{mM} \mathrm{cm}^{-1} \cdot \mathrm{l}^{-1}\right)$ using the Beer's Law.

\section{Results and Discussion}

\subsection{Measuring Lipid Peroxidation}

Malondialdehyde (MDA), a major degradation product of lipid hydroperoxides, has attracted much attention as a marker for assessing the extent of lipid peroxidation [29] [30]. The compound is of particular concern since it has been shown to be mutagenic and carcinogenic and implicated in other pathological processes such as the formation of fluorescent pigments typical of cellular aging.

The most common method for measuring MDA in food products and biological samples seems to be the thiobarbituric acid (TBA) test, which is based on spectrophotometric quantitation of the pink complex formed after reaction of MDA with two molecules of TBA [31] [32]. The TBA test can be performed (a) by directly heating the sample with TBA followed by separation of the pink complex produced (b) by distillation of the sample followed by reaction of the distillate with TBA (c) by extraction of the lipid portion of the sample with organic solvents and reaction of the extract with TBA and (d) by extraction of 
MDA using aqueous trichloroacetic or perchloric acid and reaction with TBA.

Although the distillation method is the most frequently used procedure and may be regarded as the standard method for MDA analysis, it is more cumbersome and requires more time than the aqueous acid extraction method. Furthermore, heating during distillation enhances the degradation of existing lipid hydroperoxides and, thus, additional MDA and other TBA-reactive substances (TBARS) may be formed even in the presence of metal chelators or phenolic antioxidants. The aqueous acid extraction method is also preferred by many workers because it is simple and gives results that are highly correlated with those of distillation and sensory evaluation methods.

\subsection{Effects of TRP-TPMs Derivatives 2a-c on Lipid Peroxidation}

Figures 3-5 show the results of several doses on concentrations $(0,5,10,15,20$, $25 \mu \mathrm{M})$ of TRP-TPMs derivatives on lipid peroxidation. For compound $2 \mathrm{a}$, the

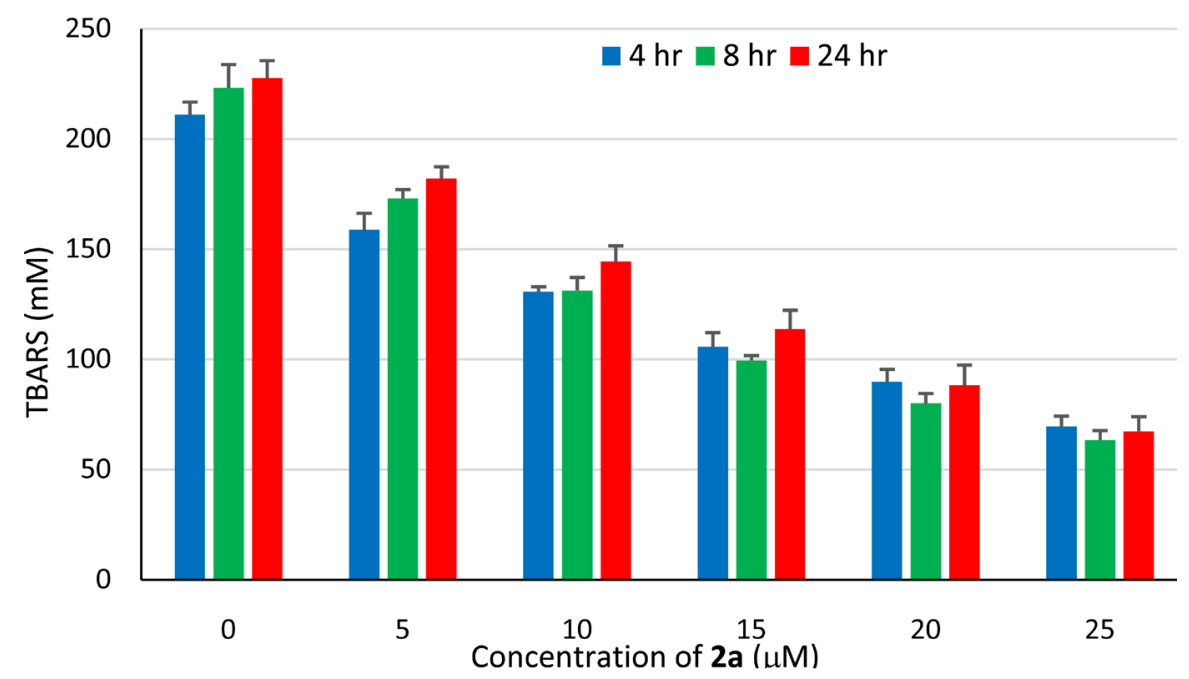

Figure 3. Effect of 2a on lipid peroxidation.

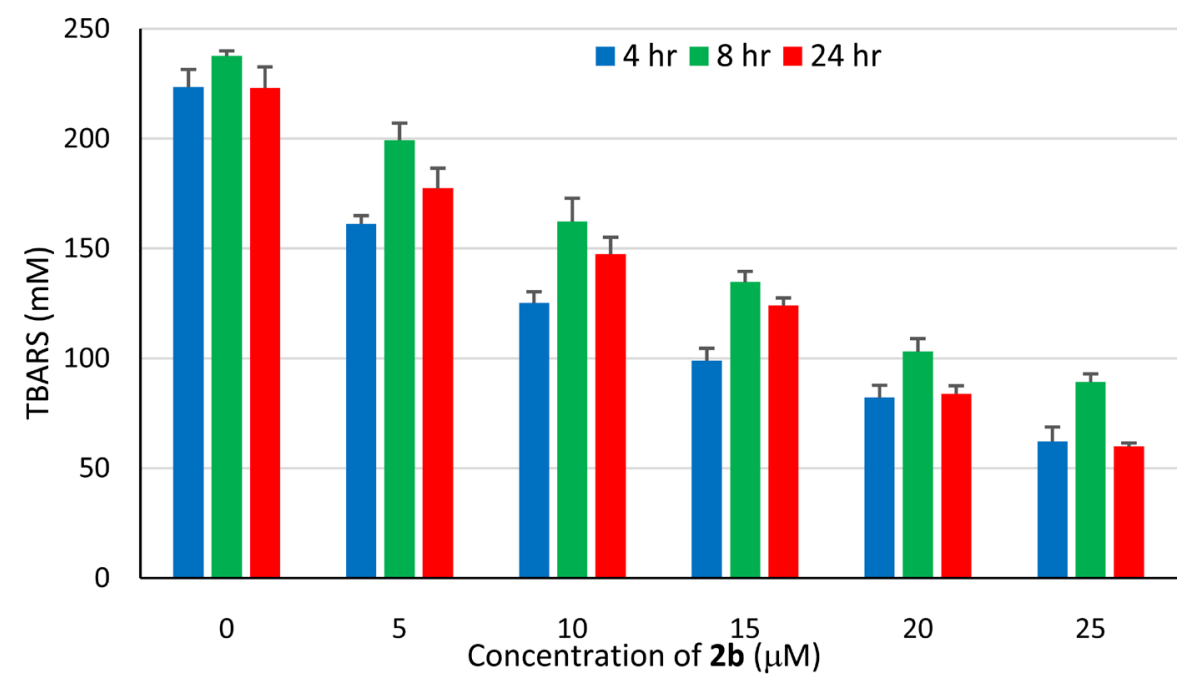

Figure 4. Effect of $2 b$ on lipid peroxidation. 
results showed a similar trend for 4,8 , and $24 \mathrm{hrs}$. of incubation. But both 8 and the $24 \mathrm{hr}$ incubations showed a remarkable decrease in lipid peroxides compared to the $4 \mathrm{hr}$ (Figure 3 ). For compound $2 \mathrm{~b}$, the results showed a similar trend for 4,8 , and $24 \mathrm{hrs}$. of incubation. However, the decrease in lipid peroxidation was more for the 24-hr compared to the 4 and $8 \mathrm{hr}$ incubation with the above drugs (Figure 4). For compound 2c, the results showed the trend for 4, 8, and $24 \mathrm{hrs}$. of incubation which indicates that the compounds $2 \mathrm{c}$ reduced lipid peroxides in a dose and time dependent manner. The reduction in lipid peroxides were greater for the $24 \mathrm{hr}$ at the high doses $(20$ and $25 \mu \mathrm{M})$ compared to the other two time periods (Figure 5).

\subsection{Effects of TPM Derivatives 1a-c on Lipid Peroxidation}

Figures 6-8 show the results of several doses on concentrations $(0,5,10,15,20$, $25 \mu \mathrm{M})$ of TPM derivatives on lipid peroxidation. A similar reduction in lipid

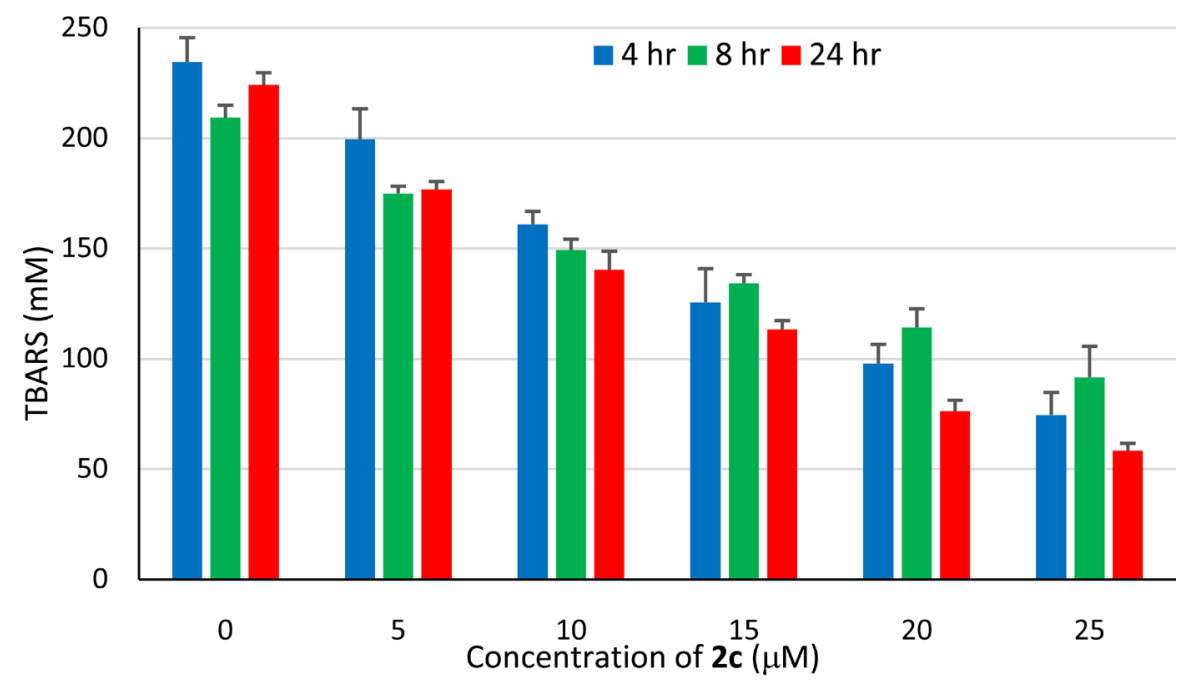

Figure 5. Effect of $2 \mathrm{c}$ on lipid peroxidation.

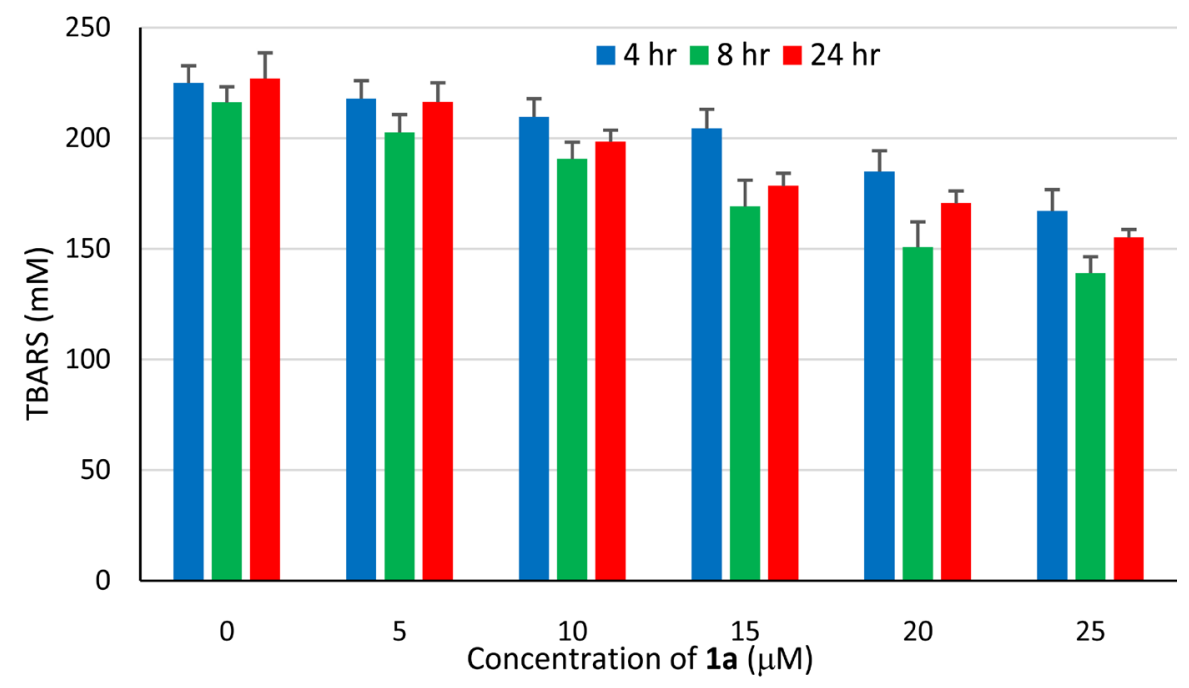

Figure 6. Effect of 1a on lipid peroxidation. 


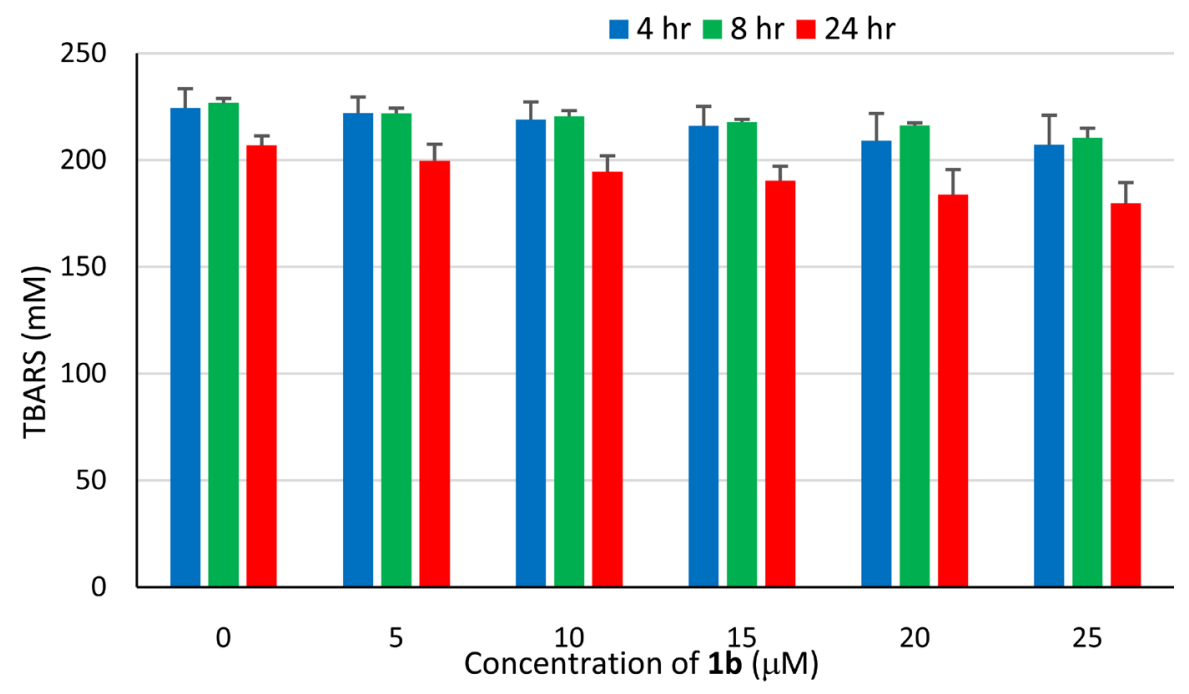

Figure 7. Effect of $1 \mathrm{~b}$ on lipid peroxidation.

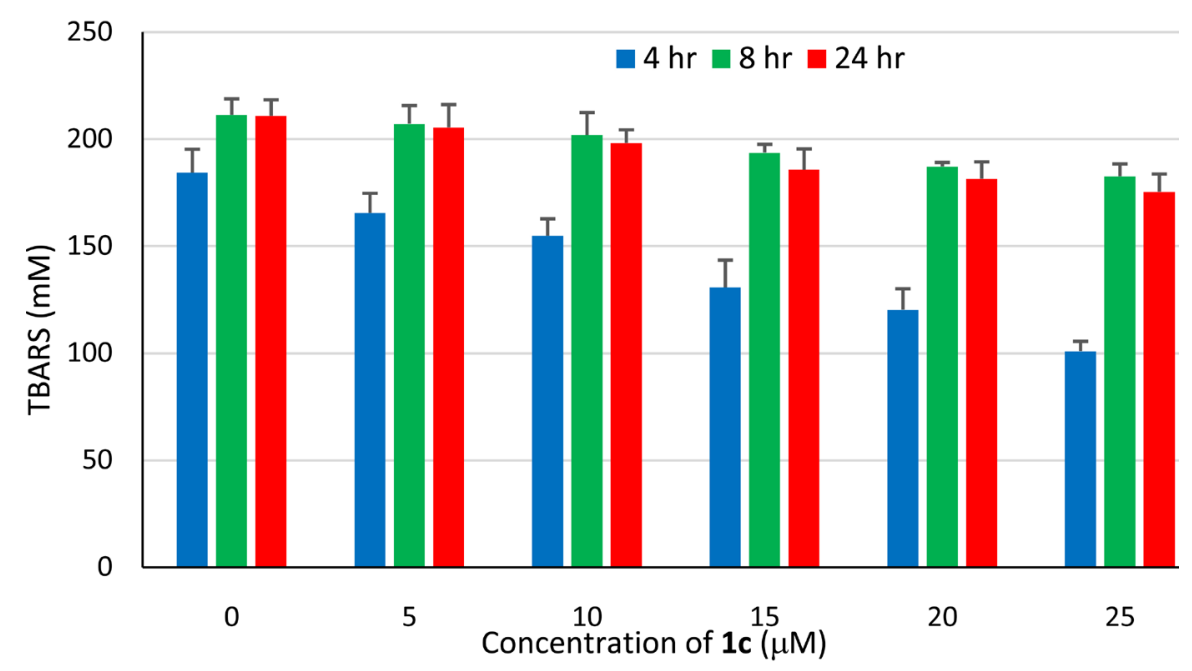

Figure 8. Effect of 1c on lipid peroxidation.

peroxidation was observed for all the 4 and $8 \mathrm{hr}$ incubation periods. The 24-hr incubation did result in the reduction of lipid peroxides for all the doses tested.

For compound 1a, there was slight decrease in lipid peroxides from 15 - 25 $\mu \mathrm{M}$ dose levels. The slight decreases for the 4 and $24 \mathrm{hr}$ incubations were not significantly different from each other (Figure 6). For compound $1 \mathrm{~b}$, a similar reduction in lipid peroxidation was observed for all the 4 and $8 \mathrm{hr}$ incubation periods. The 24-hr incubation did result in the reduction of lipid peroxides for all the doses tested (Figure 7). Finally, for compound 1c, the results indicated that the $8 \mathrm{hr}$ incubation reduced lipid peroxide compared to the 4 and $24 \mathrm{hr}$ there were no differences in lipid peroxides between the various doses for the 24 hr incubation period (Figure 8).

\subsection{Discussion}

Notwithstanding remarkable progress in understanding the pathophysiology of 
cancer and the discovery of several therapeutic prophylactics over the last few decades, cancer continues to be "the emperor of all maladies, the king of terrors". Cancer arises by accumulation of a number of mutations and epigenetic changes that endow fundamental alterations in genetic expression of a normal cell, driving it towards cancerous growth. Such cancerous growth is characterized by unregulated proliferation and tissue invasion. Years of studies have identified few specific properties of cancer cells including their metastatic property, property of unregulated cell proliferation, avoidance of apoptosis, escaping replicative senescence and avoiding differentiation, genetic instability, etc. It was therefore envisioned that modulating the lipophilicity of triptorelin might increase its cellular uptake, decrease the effect of drug efflux, thereby improving its bioefficacy and reduce the development of resistant cancer cells. Lipophilicity of triptorelin was increased by conjugating the drug with various tris (4-methoxyphenyl) methanol derivatives using long chain hydrophobic linkers. The linker used was dodecanedioic acid. The Tris (4-methoxyphenyl) Methanol were substituted at the third carbon of each of their three aromatic rings with $\mathrm{F}$, $\mathrm{NO}_{2}$, and $\mathrm{CH}_{3}$.

In vitro lipid peroxidation assays using thiobarbituric acid (TBA) method revealed that the compound $2 \mathrm{c}$ reduced lipid oxidation in a dose and time dependant manner. Interestingly there was greater reduction of the lipid oxidation during 4 - and 24 -hours incubation compared to 8 hours. The compound $2 \mathrm{~b}$ also effectively reduced the amount of lipid peroxidation in a dose and time dependant manner. Figure 4 indicates a progressive reduction in lipid peroxidation with increasing concentrations of the compound $2 \mathrm{~b}$ from $0-25 \mu \mathrm{m}$ with maximal efficacy being at 25 micromolar concentration after $24 \mathrm{hrs}$ of incubation. The results show similar trend for 4,8 , \& $24 \mathrm{hrs}$ of incubation of the compound $\mathbf{2 b}$. The decrease in lipid peroxidation, however, was more for the $24-\mathrm{hr}$ compared to the 4 and $8 \mathrm{hr}$ incubation with the above drugs. Similar to the compound $2 c$, it is difficult to estimate the maximal effective concentration of tris (4-methoxy-3-methylphenyl) methanol triptorelin from this data, since none of the incubation period indicates saturation in decrease of lipid oxidation. Studies of lipid peroxidation as depicted in Figure 8 indicate that incubation with compound $2 \mathrm{~b}$ results in a progressive reduction in lipid peroxidation with increasing concentration of the prodrug from $0-25 \mu \mathrm{m}$ with maximal efficacy being at 25 micromolar concentration after $8 \mathrm{hrs}$ of incubation. The results however show similar trend for $4,8, \& 24$ hrs of incubation. Similar to the other prodrugs, it is difficult to estimate the maximal effective concentration of tris (4-methoxy-3-methylphenyl) methanol Triptorelin from this data. The increase in efficacy at 8 hours of incubation compared to $4 \mathrm{hrs}$ and $24 \mathrm{hrs}$ might be due to the fact that the particular time point of 8 hours may represent optimal incubation period for the compound $2 \mathrm{a}$, with lesser incubation providing reduced threshold of antioxidant activity and higher incubation rendering the pro-drug to increased areal oxidation.

Unconjugated compound $1 \mathrm{a}$ by itself was only moderately efficacious in re- 
ducing lipid peroxidation. The maximum reduction was seen at the dose of 25 $\mu \mathrm{mol}$ after 24 hours incubation. The compound $\mathbf{1 b}$ was capable of significantly decreasing lipid peroxidation only at higher dosages under 24 hour's incubation. The compound $1 \mathrm{c}$ could progressively reduce lipid peroxidation under $4 \mathrm{hrs}, 8$ hrs and 24 hours incubation but the 8 -hr incubation was more effective.

\section{Conclusions}

The primary role of triptorelin conjugated pro-drug is to impart lipophilicity for increased uptake by the cell membrane. The data reported in the current studies indicate that the TPM derivatives have some antioxidant activity. This may be due to the fact that their attachment does not abrogate the antioxidant potential of triptorelin itself, and that these TPM derivatives at high concentrations also do contribute to the total antioxidant activity of the triptorelin conjugates.

It can therefore be envisioned that the triptorelin conjugates might have higher in vitro antioxidant activity than unconjugated triptorelin. Such hypothesis however remains to be tested in in vitro experiments, for example the use of several mammalian cell lines. The experimental design of such a prospective study will entail the evaluation of both the antioxidant potential of the triptorelin conjugates and unconjugated triptorelin in comparative studies. Thus, the measurement of lipid peroxides can again be used as a faithful marker in such a comparative study.

\section{Acknowledgements}

We acknowledge the financial support from the National Cancer Institute, MMC-Vanderbilt-TSU Partners in Eliminating Cancer Disparities (MVTCP), Grant Number 5U54CA163066-03. We also acknowledge the financial support from the USDA National Institute of Food and Agriculture, Grant\# TENX-1608-FS. We thank US Department of Education, Title III Part B, grant number P031B090214 for partial financial support. Jawzah Alnakhli and Samiyah Alhamed acknowledge the scholarship provided by the Saudi Arabian Cultural Mission to the US (SACM).

\section{Conflicts of Interest}

The authors declare no conflicts of interest regarding the publication of this paper.

\section{References}

[1] Thurston, B.R. (2006) Chemistry and Pharmacology of Anticancer Drugs. CRC Press, Boca Raton, 139-151.

[2] Leonard, G.G. (2009) Effective Testosterone Suppression for Prostate Cancer: Is There a Best Castration Therapy? Reviews in Urology, 11, 52-60.

[3] Yang, X., Deng, W., Fu, L., Blanco, E., Gao, J., Quan, D. and Shuai, X. (2008) Folate Functionalized Polymeric Micelles for Tumor Targeted Delivery of a Potent Multidrug-Resistance Modulator FG020326. Journal of Biomedical Materials Research 
Part A, 86, 48-60. https://doi.org/10.1002/jbm.a.31537

[4] Chavanpatil, M.D., Khdair, A., Gerard, B., Bachmeier, C., Miller, D.W., Shekhar, M.P.V. and Panvam, J. (2007) Surfactant-Polymer Nanoparticles Overcome P-Glycoprotein-Mediated Drug Efflux. Molecular Pharmacology, 4, 730-738. https://doi.org/10.1021/mp070024d

[5] Sadava, D., Coleman, A. and Kane, S.E. (2002) Liposomal Daunorubicin Overcomes Drug Resistance in Human Breast, Ovarian and Lung Carcinoma Cells. Journal of Liposome Research, 12, 301-309. https://doi.org/10.1081/LPR-120016196

[6] Ibsen, S., Zahavy, E., Wrasdilo, W., Berns, M., Chan, M. and Esener, S. (2010) A Novel Doxorubicin Prodrug with Controllable Photolysis Activation for Cancer Chemotherapy. Pharmaceutical Research, 27, 1848-1860.

https://doi.org/10.1007/s11095-010-0183-x

[7] Chhikara, B.S. and Parang, K. (2010) Development of Cytarabine Prodrugs and Delivery Systems for Leukemia Treatment. Expert Opinion on Drug Delivery, 7, 1399-1414. https://doi.org/10.1517/17425247.2010.527330

[8] Kratz, F. (2007) DOXO-EMCH (INNO-206): The First Albumin Binding Prodrug of Doxorubicin to Enter Clinical Trials. Expert Opinion on Investigational Drugs, 16, 855-866. https://doi.org/10.1517/13543784.16.6.855

[9] Wang, Y., Li, L., Jiang, W., Yang, Z. and Zhang, Z. (2006) Synthesis and Preliminary Antitumor Activity Evaluation of a DHA and Doxorubicin Conjugate. Bioorganic \& Medicinal Chemistry Letters, 16, 2974-2977. https://doi.org/10.1016/j.bmcl.2006.02.066

[10] Kumar, S.A., Peter, Y.A. and Nadeau, J.L. (2008) Facile Biosynthesis, Separation and Conjugation of Gold Nanoparticles to Doxorubicin. Nanotechnology, 19, 495-501. https://doi.org/10.1088/0957-4484/19/49/495101

[11] You, J., Zhang, G. and Li, C. (2010) Exceptionally High Payload of Doxorubicin in Hollow Gold Nanosphere for Near-Infrared Light Triggered Drug Release. ACS Nano, 4, 1033-1041. https://doi.org/10.1021/nn901181c

[12] Massing, U. and Fuxius, S. (2000) Liposomal Formulations of Anticancer Agents: Selectivity and Effectiveness. Drug Resistance Updates, 3, 171-177. https://doi.org/10.1054/drup.2000.0138

[13] Derossi, D., Joliot, A.H., Chassaing, G. and Prochiantz, A. (1994) The Third Helix of the Antennapedia Homeodomain Translocates through Biological Membranes. The Journal of Biological Chemistry, 269, 10444-10450.

[14] Derossi, D., Chassaing, G. and Prochiantz, A. (1998) Trojan Peptides: The Penetratin System for Intracellular Delivery. Trends in Cell Biology, 8, 84-87. https://doi.org/10.1016/S0962-8924(98)80017-2

[15] Meyer-Losic, F., Quinonero, J., Dubois, V., Alluis, B., Dechambre, M., Michel, M., Cailler, F., Fernandez, A.M., Trouet, A. and Kearsey, J. (2006) Improved Therapeutic Efficacy of Doxorubicin through Conjugation with a Novel Peptide Drug Delivery Technology (Vectocell). Journal of Medicinal Chemistry, 49, 6908-6916. https://doi.org/10.1021/jm0606591

[16] Che, C., Yang, G., Thiot, C., Lacoste, M.-C., Currie, J.-C., Demeule, M., Regina, A., Beliveau, R. and Castaigne, J.-P. (2010) New Angiopep Modified Doxorubicin (ANG1007) and Etoposide (ANG1009) Chemotherapeutics with Increased Brain Penetration. Journal of Medicinal Chemistry, 53, 2814-2824.

https://doi.org/10.1021/jm9016637

[17] Lindgren, M., Rosenthal-Aizman, K., Saar, K., Eiriksdottir, E., Jiang, Y., Sassian, M., 
Ostlund, P., Hallbrink, M. and Langel, U. (2006) Overcoming Methotrexate Resistance in Breast Cancer Tumour Cells by the Use of a New Cell-Penetrating Peptide. Biochemical Pharmacology, 71, 416-425. https://doi.org/10.1016/j.bcp.2005.10.048

[18] Amir, N.S., Rakesh, T., Bhupender, S.C., Dindyal, M. and Keykavous, P. (2013) Design and Biological Evaluation of Cell-Penetrating Peptide-Doxorubicin Conjugates as Prodrugs. Molecular Pharmaceutics, 10, 488-499.

https://doi.org/10.1021/mp3004034

[19] Zhu, S., Hong, M., Zhang, L., Tang, G., Jiang, Y. and Pei, Y. (2010) PEGylated PAMAM Dendrimer-Doxorubicin Conjugates: In Vitro Evaluation and in Vivo Tumor Accumulation. Pharmaceutical Research, 27, 161-174. https://doi.org/10.1007/s11095-009-9992-1

[20] Scalbert, A., Manach, C., Morand, C. and Remesy, C. (2005) Dietary Polyphenols and the Prevention of Diseases. Critical Reviews in Food Science and Nutrition, 45, 287-306. https://doi.org/10.1080/1040869059096

[21] Spencer, J.P., Abd El Mohsen, M.M., Minihane, A.M. and Mathers, J.C. (2008) Biomarkers of the Intake of Dietary Polyphenols: Strengths, Limitations and Application in Nutrition Research. British Journal of Nutrition, 99, 12-22. https://doi.org/10.1017/S0007114507798938

[22] Bhooshan, P.K. and Rizv, S.I. (2009) Plant Polyphenols as Dietary Antioxidants in Human Health and Disease. Oxidative Medicine and Cellular Longevity, 2, 270-278. https://doi.org/10.4161/oxim.2.5.9498

[23] Yang, C.S., Landau, J.M., Huang, M.T. and Newmark, H.L. (2001) Inhibition of Carcinogenesis by Dietary Polyphenolic Compounds. Annual Review of Nutrition, 21, 381-406. https://doi.org/10.1146/annurev.nutr.21.1.381

[24] Johnson, I.T., Williamson, G. and Musk, S.R.R. (1994) Anticarcinogenic Factors in Plant Foods: A New Class of Nutrients? Nutrition Research Reviews, 7, 175-204. https://doi.org/10.1079/NRR19940011

[25] Talalay, P., De Long, M.J. and Prochaska, H.J. (1988) Identification of a Common Chemical Signal Regulating the Induction of Enzymes That Protect against Chemical Carcinogenesis. Proceedings of the National Academy of Sciences of the United States of America, 85, 8261-8265. https://doi.org/10.1073/pnas.85.21.8261

[26] Khan, N. and Mukhtar, H. (2008) Multitargeted Therapy of Cancer by Green Tea Polyphenols. Cancer Letters, 269, 269-280. https://doi.org/10.1016/j.canlet.2008.04.014

[27] Suja, S., Bharat Raj, B., Kyung Ja, C., Keun-Hyeung, L. and Hyeongjin, C. (2007) Methylenedisalicylic Acid Derivatives: New PTP1B Inhibitors That Confer Resistance to Diet-Induced Obesity. Bioorganic \& Medicinal Chemistry Letters, 17, 2760-2764. https://doi.org/10.1016/j.bmcl.2007.02.069

[28] Mark, C., Suseela Erik, D.C., Dominique, S., Mark, E.G. and Julie, A.B. (1991) Synthesis and Anti-HIV Activities of Low Molecular Weight Aurintricarboxylic Acid Fragments and Related Compounds. Journal of Medicinal Chemistry, 34, 337-342. https://doi.org/10.1021/jm00105a053

[29] Gutteridge, J.M. and Halliwell, B. (1990) The Measurement and Mechanism of Lipid Peroxidation in Biological Systems. Trends in Biochemical Sciences, 15, 129-135. https://doi.org/10.1016/0968-0004(90)90206-Q

[30] Requena, J.R., et al. (1997) Quantification of Malondialdehyde and 4-Hydroxynonenal Adducts to Lysine Residues in Native and Oxidized Human Low-Density Lipoprotein. Biochemical Journal, 322, 317-325. https://doi.org/10.1042/bj3220317 
[31] Janero, D.R. (1990) Malondialdehyde and Thiobarbituric Acid-Reactivity as Diagnostic Indices of Lipid Peroxidation and Peroxidative Tissue Injury. Free Radical Biology \& Medicine, 9, 515-540. https://doi.org/10.1016/0891-5849(90)90131-2

[32] Gasparovic, A.C., et al. (2013) Assays for the Measurement of Lipid Peroxidation. Methods in Molecular Biology, 965, 283-296.

https://doi.org/10.1007/978-1-62703-239-1 19

\section{Abbreviations}

TBARS, thiobarbituric reactive substance

TRP, Triptorelin

TPMs, Triphenylmethanols

DCM, dichloromethane

NMP, (N-Methyl-2-pyrrolidone)

HBTU, 2-(1H-benzotriazol-1-yl)-1,1,3,3-tetramethyl uronium hexafluorophosphate

DIC, $N, N$ '-Diisopropylcarbodiimide

DIPEA, $N, N$-Diisopropylethylamine

$\mathrm{GnRH}$, gonadotropin-releasing hormone

FSH, Follicle-stimulating hormone

LH, Luteinizing hormone

LHRH, luteinizing hormone-releasing hormone. 